\title{
Original Article Serum alkaline phosphatase may play a role in the differential diagnosis of sarcoidosis and tuberculosis
}

\author{
Seda Tural Onur ${ }^{1}$, Sinem Iliaz², Raim Iliaz ${ }^{3}$, Sinem Sokucu³, Cengiz Ozdemir ${ }^{3}$ \\ ${ }^{1}$ Department of Pulmonary Medicine, Edikule Chest Diseases and Thoracic Surgery Training and Research \\ Hospital, Istanbul, Turkey; ${ }^{2}$ Department of Pulmonary Medicine, Koç University Hospital, Istanbul, Turkey; \\ ${ }^{3}$ Department of Gastroenterology, Istanbul Faculty of Medicine, Istanbul University, Istanbul, Turkey
}

Received January 12, 2016; Accepted May 20, 2016; Epub July 15, 2016; Published July 30, 2016

\begin{abstract}
Background: Reaching the differential diagnosis of tuberculosis and sarcoidosis can be difficult due to granulomatous inflammation. The aim of this study was to determine alkaline phosphatase (ALP) activity in serum for the differential diagnosis of tuberculosis and sarcoidosis. Methods: This study comprised 242 subjects: 105 acid-fast bacilli (AFB) positive and/or culture-positive patients with pulmonary tuberculosis, 90 patients with biopsyproven sarcoidosis and a control group consisting of 47 healthy controls were included. ALP activity was measured in serum at the first admission of the patients. Results: The mean serum ALP was $112.74 \pm 55.14 \mathrm{IU} / \mathrm{L}$ in pulmonary tuberculosis, $76.14 \pm 34.23 \mathrm{IU} / \mathrm{L}$ in sarcoidosis, and $66.87 \pm 18.49 \mathrm{IU} / \mathrm{L}$ in the control group, respectively. There was a statistically significant difference between the patient population and the control group $(P=0.03)$. Also, there was a statistically significant difference between the tuberculosis and sarcoidosis groups $(P=0.034)$. According to the comparison of tuberculosis and sarcoidosis, the cut-off value was determined as $71.50 \mathrm{IU} / \mathrm{L}$, which had sensitivity of $80 \%$, specificity of $51 \%$, PPV of $66 \%$, NPV of $69 \%$, accuracy of $67 \%$, and the AUC was 0.728 . Conclusion: ALP, as a little-known marker for tuberculosis and sarcoidosis, was significantly increased in the pulmonary tuberculosis group compared with the sarcoidosis group. As such, it may be a useful tool for the differentiation of tuberculosis and sarcoidosis.
\end{abstract}

Keywords: Alkaline phosphatase, diagnostic challenges, tuberculosis

\section{Introduction}

Tuberculosis is an infectious disease that caused death of 1.5 million people in 2014 [1]. It has an important role among pulmonary and extrapulmonary granulomatous diseases. Its incidence is steadily increasing because of the association of tuberculosis with AIDS. So, physicians experience difficulties in the differential diagnosis and treatment of tuberculosis. To achieve a definite diagnosis of tuberculosis, Mycobacterium tuberculosis bacilli must be detected in sputum [1]. However, this is not always possible. The differential diagnosis of sarcoidosis leads to some difficulties because lung and lymph node involvement mimics tuberculosis. Also, both cause granulomatous inflammation. Therefore, in order to achieve the differential diagnosis between tuberculosis and sarcoidosis more easily, new methods are being developed [2-4].
Alkaline phosphatase (ALP) hydrolyzes phosphate esters at $\mathrm{pH}$ 9. The role of ALP has been investigated in other organ systems and their diseases [5, 6]. The importance of alkaline phosphatase in respiratory medicine was studied previously in a limited number of studies involving tuberculous pleurisy $[7,8]$.

For the differential diagnosis of sarcoidosis and tuberculosis, minimally invasive methods are being researched. We planned this study to evaluate the use of serum ALP in the differential diagnosis of tuberculosis and sarcoidosis, compared with healthy controls.

\section{Materials and methods}

In our study, 105 patients with bacteriologically-proven pulmonary tuberculosis, 90 patients with biopsy-proven sarcoidosis, and a control group consisting of 47 healthy patients were 
Table 1. Characteristics of the groups

\begin{tabular}{lcccc}
\hline $\begin{array}{l}\text { Parameters } \\
\text { Mean } \pm \text { SD or } \\
\mathrm{n} / \%\end{array}$ & $\begin{array}{c}\text { Sarcoidosis } \\
\mathrm{N}=90\end{array}$ & $\begin{array}{c}\text { Tuberculosis } \\
\mathrm{N}=105\end{array}$ & $\begin{array}{c}\text { Control } \\
\mathrm{N}=47\end{array}$ & $P$ value \\
\hline Sex (male) & $21 / 23.3 \%$ & $51 / 48.6 \%$ & $18 / 38.3 \%$ & 0.001 \\
Age (years) & $44.3 \pm 11.2$ & $42.3 \pm 19.4$ & $43.7 \pm 13.4$ & 0.67 \\
ALP (IU/L) & $76.1 \pm 34.2$ & $112.7 \pm 55.1$ & $66.8 \pm 18.4$ & $<0.001$ \\
CRP $(\mathrm{mg} / \mathrm{L})$ & $6.8 \pm 7.1$ & $98.2 \pm 72.1$ & $2.4 \pm 2.5$ & $<0.001$ \\
ESR $(\mathrm{mm} / \mathrm{st})$ & $19.2 \pm 18.5$ & $63.5 \pm 28.9$ & $13.1 \pm 9.1$ & $<0.001$ \\
WBC $(\mathrm{K} / \mathrm{uL})$ & $6.9 \pm 1.7$ & $10.2 \pm 3.4$ & $7 \pm 1.4$ & $<0.001$ \\
\hline
\end{tabular}

ALP: alkaline phosphatase, CRP: $\mathrm{C}$ reactive protein, ESR:erythrocyte sedimentation rate, WBC: white blood cell, $\mathrm{P}<0.05$ is accepted significant.

included. Sputum analyses of all patients with tuberculosis were acid-fast bacilli (AFB)-positive and/or culture-positive. We included asymptomatic patients who were admitted to the internal medicine out-patient clinic for routine health checks for the control group. We excluded patients with known osteoporosis, cholestatic liver disease, and any malignancy.

The study design was retrospective data analysis. Between June 2011 and June 2014, a total of 368 patients' data were analyzed retrospectively. Inclusion criteria were definite diagnosis of tuberculosis or sarcoidosis, available archive data (biochemical values), absence of any other organ malignancies, absence of any disease causing increase in ALP (e.g., chronic liver disease, osteoporosis, osteomalacia), not receiving immune suppressive treatment.

Medical history and findings in physical examination were recorded. The complete blood count (CBC) taken at time of the first admission was used and analyzed using an Abbott CellDyne 3700 System (Abbott Diagnostics, Santa Clara, CA, USA). Also, serum biochemical parameters taken at time of the first admission was used. Serum biochemical parameters were measured using a Beckman Coulter AU 2700 plus (Olympus, Tokyo, Japan).

The study was approved by the Institutional Ethics Committee (number: 3974; date: 22.06. 2015). The study conformed to the ethical guidelines of the 2000 Helsinki declaration.

\section{Statistical analysis}

Statistical analysis was performed using Statistical Package for Social Sciences (SPSS) version 22 (Armonk, NY: IBM Corp) software.
Descriptive values were given as mean and standard deviation. Categorical variables were expressed as the number of cases and the percentage value. Kolmogorov-Smirnov and Shapiro-Wilk tests were used to determine whether continuous variables were normally distributed. In the comparison of groups, Student's t-test and Mann-Whitney $U$ test were used according to the normality of distribution of the variables. The comparison of categorical variables was performed using Chi-square and Fisher's exact tests. $P$ value less than 0.05 were considered statistically significant. Receiver operating characteristics (ROC) curves and areas under the ROC curves (AUC) with 95\% confidence intervals (Cl) were calculated for the criteria to evaluate optimum cut-off points. In addition to using cut-off points derived from ROC curves, the utility of each criterion for identifying pulmonary tuberculosis was evaluated by calculating the sensitivity, specificity, positive predictive value, negative predictive value, and accuracy.

\section{Results}

In the tuberculosis group, 54 patients were women $(51.4 \%)$ and 51 men (48.6\%) with a mean age of $42.3 \pm 19.4$ years. In the sarcoidosis group, 69 patients were women (76.7\%) and 21 men (23.3\%), with a mean age of $44.3 \pm 11.2$ years. In the control group, 29 individuals were women (61.7\%) and 18 men (38.3\%); the mean age was $43.7 \pm 13.4$ years. The study population was similar in terms of age in all groups $(P=0.67)$. Characteristics of the groups were given in Table 1.

In the group of subjects with pulmonary tuberculosis, the mean serum ALP was significantly higher compared with the healthy control subjects $(P=0.034)$. In the comparison of tuberculosis with controls, a cut-off value of $64.50 \mathrm{IU} / \mathrm{L}$ was determined in the ROC analysis. This cutoff value of ALP had sensitivity of $89 \%$, specificity of $51 \%$, positive predictive value (PPV) of $80 \%$, negative predictive value (NPV) of $67 \%$, accuracy $77 \%$ to diagnose tuberculosis. The area under the curve (AUC) was 0.823. The ROC curve is shown in Figure 1.

When the sarcoidosis and control groups were compared, serum ALP was significantly higher 


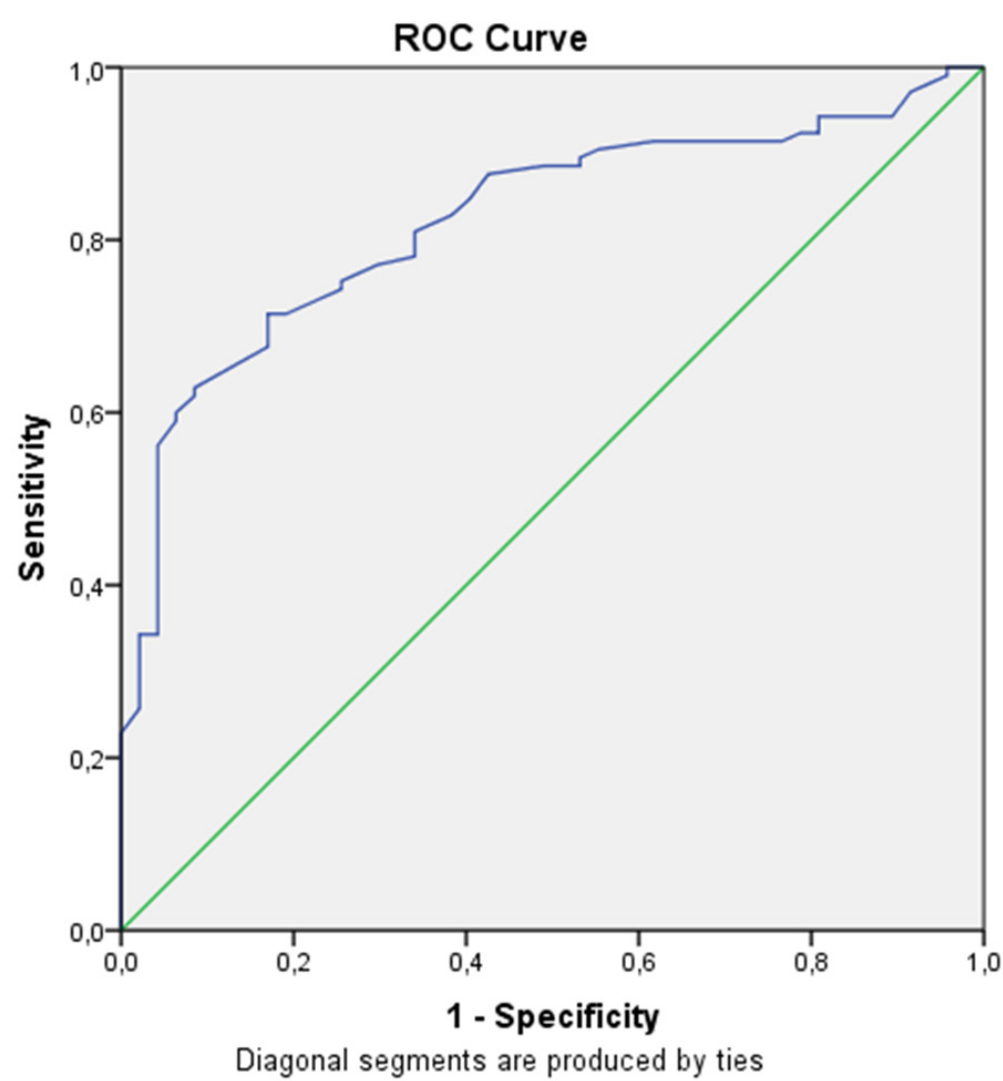

Figure 1. ROC curves comparing pulmonary tuberculosis and the control group. For serum ALP, the cut-off value of $64.50 \mathrm{IU} / \mathrm{L}$ had sensitivity of $89 \%$, specificity of $51 \%$, positive predictive value (PPV) of $80 \%$, negative predictive value (NPV) of $67 \%$, accuracy of $77 \%$, and area under the curve (AUC) was 0.823 to diagnose tuberculosis.

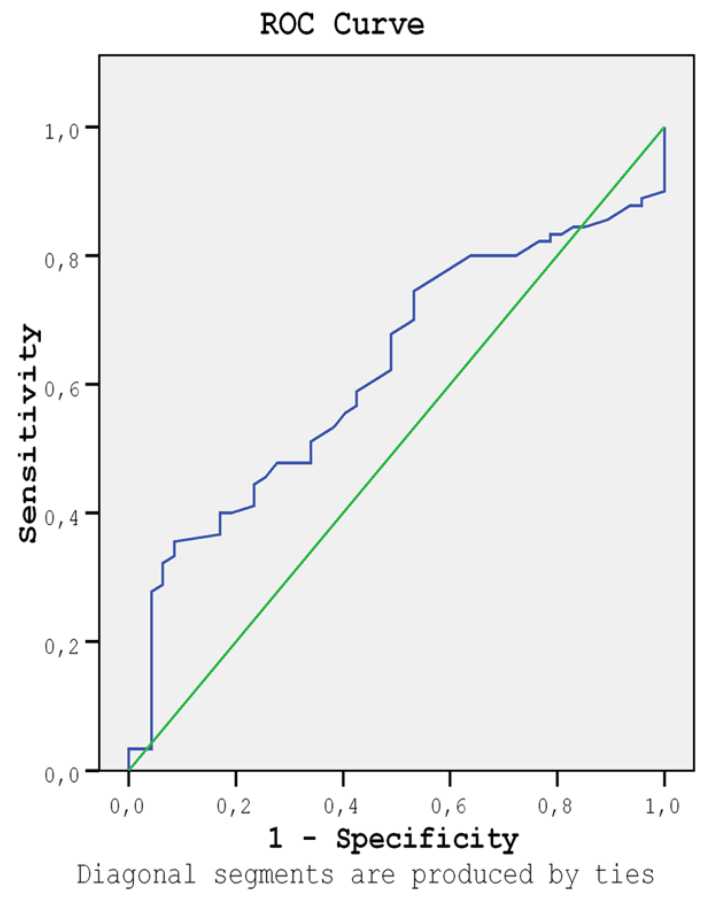

Figure 2. ROC curves comparing the sarcoidosis and control group. Serum ALP cut-off value of $63.5 \mathrm{IU} / \mathrm{L}$ had sensitivity of $68 \%$, specificity of $52 \%$, PPV of $73 \%$, NPV of $45 \%$, accuracy of $62 \%$, and AUC was 0.371.

in the sarcoidosis group $(P=$ 0.049). Among these groups, the cut-off value of $63.5 \mathrm{IU} / \mathrm{L}$ was determined to diagnose sarcoidosis. For this cut-off value of ALP, we found sensitivity of $68 \%$, specificity of $52 \%$, PPV of $73 \%$, NPV of $45 \%$, accuracy of $62 \%$, and AUC was 0.371. The ROC curve is presented in Figure 2.

Serum ALP was significantly higher in the tuberculosis group compared with the sarcoidosis group $(P=0.034)$. According to the comparison of tuberculosis and sarcoidosis, the cut-off value of $71.50 \mathrm{IU} / \mathrm{L}$ was determined. For this cut-off value of ALP, there was sensitivity of $80 \%$, specificity of $51 \%$, PPV of $66 \%$, NPV of $69 \%$, accuracy of $67 \%$. AUC was 0.728 . The ROC curve is shown in Figure 3. We also gave the ROC analysis results for CRP, ESR, and white blood cell count (WBC) to discriminate tuberculosis from sarcoidosis in Table 2.

In the correlation analysis, CRP had significant positive correlation with ALP in sarcoidosis group ( $P=0.008, r=0.289$ ). In tuberculosis group, ALP had significant positive correlation with CRP and WBC $(P<0.001, r=0.378$ and $P=0.002, r=0.301$, respectively). There were no such correlation for these parameters in the control group.

\section{Discussion}

The differentiation of sarcoidosis and tuberculosis is sometimes challenging because they share similar clinical presentations and insufficient clinical evidence. The results of our study showed that serum ALP was a significantly dis- 


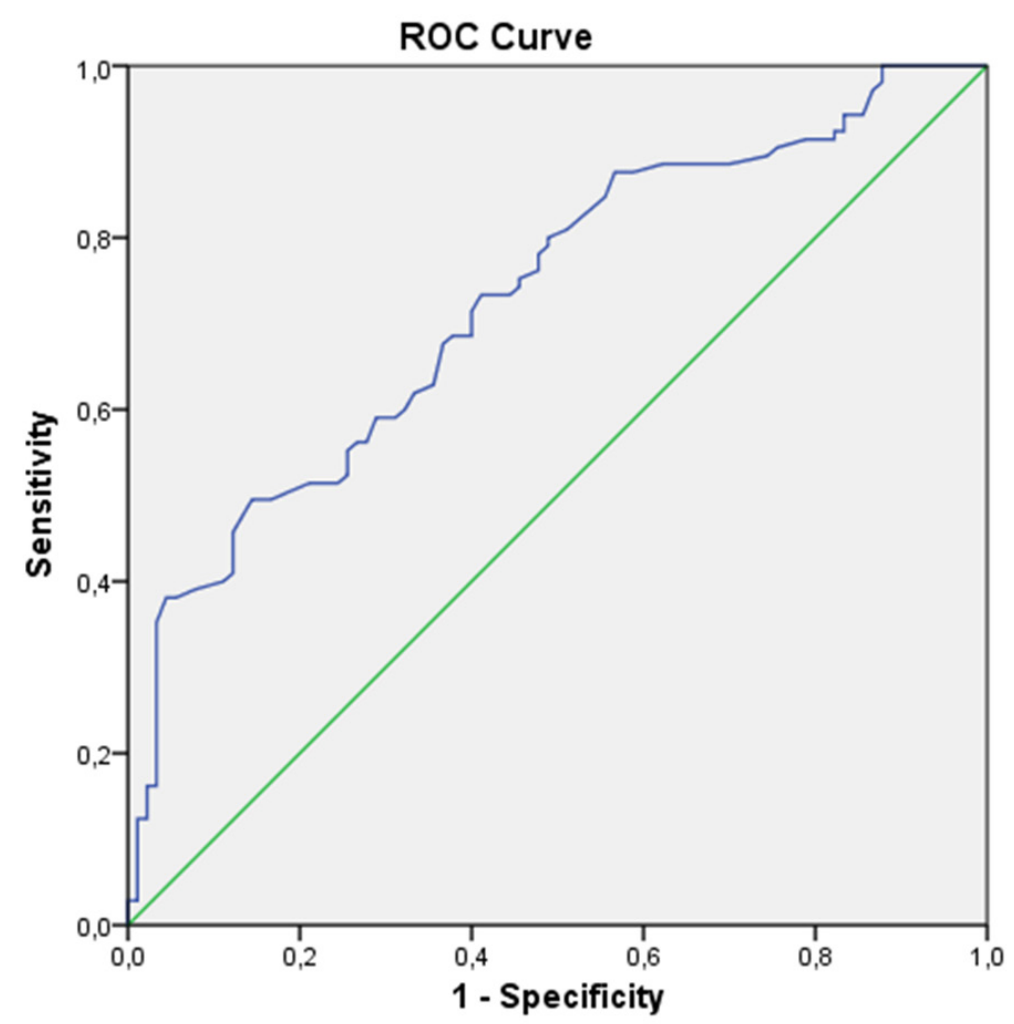

Diagonal segments are produced by ties

Figure 3. ROC curves comparing pulmonary tuberculosis and sarcoidosis. For the serum ALP cut-off value of $71.50 \mathrm{IU} / \mathrm{L}$, there was sensitivity of $80 \%$, specificity of $51 \%$, PPV of $66 \%$, NPV of $69 \%$, accuracy of $67 \%$ and AUC was 0.728 .

Table 2. ROC analysis results for biomarkers to discriminate tuberculosis from sarcoidosis

\begin{tabular}{lccccccc}
\hline Biomarkers & Cut-off & Sensitivity & Specificity & PPV & NPV & Accuracy & AUC \\
\hline ALP (IU/L) & 71.5 & $80 \%$ & $51 \%$ & $66 \%$ & $69 \%$ & $67 \%$ & 0.728 \\
CRP (mg/L) & 19 & $91 \%$ & $91 \%$ & $92 \%$ & $86 \%$ & $89 \%$ & 0.965 \\
ESR (mm/st) & 34.5 & $86 \%$ & $85 \%$ & $84 \%$ & $88 \%$ & $86 \%$ & 0.870 \\
WBC (K/uL) & 7.77 & $81 \%$ & $78 \%$ & $79 \%$ & $76 \%$ & $77 \%$ & 0.832 \\
\hline
\end{tabular}

ALP: alkaline phosphatase, CRP: C reactive protein, ESR: erythrocyte sedimentation rate, WBC: white blood cell, PPD: positive predictive value, NPV: negative predictive value, AUC: area under the curve. phy (EBUS). The authors reported that heterogeneous echotexture or coagulation necrosis in the lymph nodes evaluated using EBUS were specific for tuberculosis involvement and could differentiate tuberculosis from sarcoidosis [10].

Previously, a few researchers tried to contribute to the differential diagnosis of tuberculosis with ALP. Jadhav et al. investigated the activity of ALP and adenosine deaminase (ADA) in sputum as a noninvasive method and both parameters were proved to contribute to the differential diagnosis [11]. Our study demonstrated the value of ALP in tuberculosis with a larger number of cases.

ALP is produced by type II pneumocytes in the lungs. Capelli et al. studied ALP in interstitial lung diseases. They showed that, especially in patients with sarcoidosis with Stage 2 and 3/4 disease, ALP increased significantly in bronchooalveolar lavage (BAL), but could not get enough availability in clinical practise [12]. Unlike the studies with respiratory samples, our study showed that serum ALP contributed to the differential diagnosis of tuberculosis and it was a minimally invasive method. tinctive feature in the differential diagnosis of tuberculosis with parenchymal involvement and sarcoidosis. Researchers are still investigating many invasive or non-invasive methods to support the distinction between these two granulomatous diseases. Recently, Du and colleagues proved that intercellular adhesion molecule-1 (ICAM-1) and leptin were potential markers for the differential diagnosis of sarcoidosis and sputum-negative tuberculosis [9]. Dhooria et al. demonstrated sonographic features of lymph nodes involved with tuberculosis or sarcoidosis on endobronchial ultrasonogra-
The mechanism of ALP increase has yet to be clearly defined in a variety of forms of tuberculosis. Metintas and colleagues investigated the differentiation of exudative pleural effusion with transudative effusion in a multiparametric study. Pleural ALP determined exudative effusion with a sensitivity of $82 \%$ and specificity of $76 \%$, despite its lower contribution to diagnosis [8]. Similarly, Jadhav et al. proved that pleural ALP made the differential diagnosis of tuberculous and non-tuberculous pleural effusions with a sensitivity of $90 \%$ and specificity of $80 \%$ [13]. In contrast, Mushtaq showed that ALP had 
no significance in differentiating exudative pleurisy with transudative pleurisy [14]. Our study showed that serum ALP could be used to differentiate tuberculosis from healthy patients and patients with sarcoidosis with high sensitivity. The overlapping clinical manifestations of granulomatous diseases can make differentiation even harder, especially when seen associated diseases such as AIDS; it is not easy to reach a definitive diagnosis and physicians need additional supporting evidence.

The mechanism of ALP cannot be fully understood because there is a limited number of studies in the literature; however, it seems important for the differential diagnosis of sarcoidosis and tuberculosis. For complicated cases, taking blood at first admission for ALP analysis seems minimally invasive, fast, easily accessible, and inexpensive. We think it is a very valuable test. A prospective case series with a larger population could increase the significance of ALP.

\section{Acknowledgements}

We thank David F. Chapman for English-language editing.

\section{Disclosure of conflict of interest}

None.

Address correspondence to: Dr. Seda Tural Onur, Department of Pulmonary Medicine, Yedikule Chest Diseases and Thoracic Surgery Training and Research Hospital, Chest Disease, Istanbul 34760, Turkey. Tel: +902124090202; Fax: +902125472233; E-mail: sedatural@yahoo.com

\section{References}

[1] WHO (World Health Organization). Global tuberculosis report 2015.

[2] Yew WW, Leung CC. Update in Tuberculosis 2005. Am J Respir Crit Med 2006; 173: 491498.

[3] Chen T, Lin J, Wang W, Fleming J, Chen L, Wang Y, Li H, Guo H, Zhou J, Chen X, Chen Y, Liao Q, Shu Y, Tan Y, Yu M, Li G, Zhou L, Zhong Q, Bi L, Guo L, Zhao M. Cytokine and Antibody Based Diagnostic Algorithms for Sputum CulturePositive Pulmonary Tuberculosis. PLoS One 2015; 10: e0144705.
[4] Iliaz S, Iliaz R, Ortakoylu G, Bahadir A, Bagci BA, Caglar E. Value of neutrophil/lymphocyte ratio in the differential diagnosis of sarcoidosis and tuberculosis. Ann Thorac Med 2014; 9: 232-235.

[5] Cunha BA, Petelin A. Fever of unknown origin (FUO) due to large B-cell lymphoma: the diagnostic significance of highly elevated alkaline phosphatase and serum ferritin levels. Heart Lung 2013; 42: 67-71.

[6] Yamazoe M, Mizuno A, Nishi Y, Niwa K, Isobe $M$. Serum alkaline phosphatase as a predictor of worsening renal function in patients with acute decompensated heart failure. J Cardiol 2016; 67: 412-7.

[7] Syabbalo NC. Use of pleural alkaline phosphatase content to diagnose tuberculous effusions. Chest 1991; 99: 522-523.

[8] Metintas M, Alatas O, Alatas F, Colak O, Ozdemir N, Erginal S. Comparative analysis of biochemical parameters for differentiation of pleural exudates from transudates: Light's criteria, cholesterol, bilirubin, albumin gradient, alkaline phosphatase, creatine kinase and uric acid. Clin Chim Acta 1997; 264: 149-162.

[9] Du SS, Zhao MM, Zhang Y, Zhang P, Hu Y, Wang LS, Zhou Y, Li QH, Li Y, Du YK, He X, Li N, Yin ZF, Wei YR, Weng D, Li HP. Screening for Differentially Expressed Proteins Relevant to the Differential Diagnosis of Sarcoidosis and Tuberculosis. PLoS One 2015; 10: e0132466.

[10] Dhooria S, Agarwal R, Aggarwal AN, Bal A, Gupta N, Gupta D. Differentiating tuberculosis from sarcoidosis by sonographic characteristics of lymph nodes on endobronchial ultrasonography: a study of 165 patients. J Thorac Cardiovasc Surg 2014; 148: 662-667.

[11] Jadhav AA, Jain A. Sputum adenosine deaminase and alkaline phosphatase activity in pulmonary tuberculosis. Arch Physiol Biochem 2012; 118: 6-9.

[12] Capelli A, Lusuardi M, Cerutti CG, Donner CF. Lung alkaline phosphatase as a marker of fibrosis in chronic interstitial disorders. Am J Respir Crit Care Med 1997; 155: 249-253.

[13] Jadhav AA, Bardapurkar JS, Jain A. Alkaline phosphatase: Distinguishing between tuberculous and nontuberculous pleural effusion. Lung India 2009; 26: 77-80.

[14] Lone MA, Wahid A, Saleem SM, Koul P, Dhobi GN, Shahnawaz A. Alkaline phosphatase in pleural effusions. Indian J Chest Dis Allied Sci 2003; 45: 161-163. 